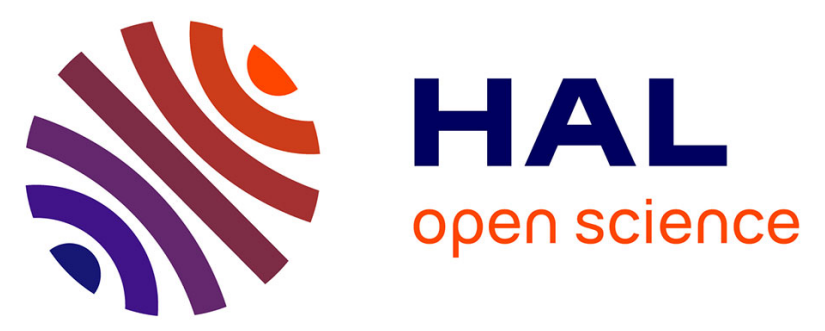

\title{
Generation of Synthetic but Visually Realistic Time Series of Cardiac Images Combining a Biophysical Model and Clinical Images
}

\author{
A. Prakosa, Maxime Sermesant, H. Delingette, S. Marchesseau, E. Saloux, \\ Pascal Allain, N. Villain, Nicholas Ayache
}

\section{To cite this version:}

A. Prakosa, Maxime Sermesant, H. Delingette, S. Marchesseau, E. Saloux, et al.. Generation of Synthetic but Visually Realistic Time Series of Cardiac Images Combining a Biophysical Model and Clinical Images. IEEE Transactions on Medical Imaging, 2013, 32 (1), pp.99-109. 10.1109/TMI.2012.2220375 . hal-00813861

\section{HAL Id: hal-00813861 https://hal.inria.fr/hal-00813861}

Submitted on 3 Dec 2013

HAL is a multi-disciplinary open access archive for the deposit and dissemination of scientific research documents, whether they are published or not. The documents may come from teaching and research institutions in France or abroad, or from public or private research centers.
L'archive ouverte pluridisciplinaire $\mathbf{H A L}$, est destinée au dépôt et à la diffusion de documents scientifiques de niveau recherche, publiés ou non, émanant des établissements d'enseignement et de recherche français ou étrangers, des laboratoires publics ou privés. 


\title{
Generation of Synthetic but Visually Realistic Time Series of Cardiac Images Combining a Biophysical Model and Clinical Images
}

\author{
Adityo Prakosa, Maxime Sermesant, Hervé Delingette, Stéphanie Marchesseau, Eric Saloux, Pascal Allain, \\ Nicolas Villain, and Nicholas Ayache
}

\begin{abstract}
We propose a new approach for the generation of synthetic but visually realistic time series of cardiac images based on an electromechanical model of the heart and real clinical 4D image sequences. This is achieved by combining three steps. The first step is the simulation of a cardiac motion using an electromechanical model of the heart and the segmentation of the end diastolic image of a cardiac sequence. We use biophysical parameters related to the desired condition of the simulated subject. The second step extracts the cardiac motion from the real sequence using non-rigid image registration. Finally, a synthetic time series of cardiac images corresponding to the simulated motion is generated in the third step by combining the motion estimated by image registration and the simulated one. With this approach, image processing algorithms can be evaluated as we know the ground-truth motion underlying the image sequence. Moreover, databases of visually realistic images of controls and patients can be generated for which the underlying cardiac motion and some biophysical parameters are known. Such databases can open new avenues for machine learning approaches.
\end{abstract}

Index Terms-synthetic 4D cardiac sequences, cardiac electromechanical model, non-rigid registration.

\section{INTRODUCTION}

D IAGNOSIS and therapy planning of cardiovascular diseases are often much improved by the analysis of the dynamic cardiac function. The advances in medical imaging offer increasingly detailed visual information on the cardiac motion. However there is a lack of quantification tools and of methods to validate them. The manual ground-truth generated by experts, which is typically used to validate segmentation algorithms, cannot be extended to motion tracking. Experts can only manually track a few landmarks, and doing this in sequences of volumetric images is very tedious, provides very limited accuracy and is prone to inter and intra-expert variabilities. In this article, we propose a framework to generate both cardiac ground-truth motion and corresponding synthetic but visually realistic images in order to enable such validation.

Copyright (c) 2012 IEEE. Personal use of this material is permitted. However, permission to use this material for any other purposes must be obtained from the IEEE by sending a request to pubs-permissions@ieee.org.

A. Prakosa, M. Sermesant, H. Delingette, S. Marchesseau and N. Ayache are with the Asclepios Research Project, Inria Sophia Antipolis, 2004 route des Lucioles - BP 93, 06902, Sophia Antipolis, France

E. Saloux is with the Cardiology, Caen University Hospital, Avenue Georges Clemenceau, 14000, Caen, France

P. Allain and N. Villain are with the Medisys Research Lab, Philips Healthcare France, 33 rue de Verdun - BP313, Suresnes, France
There are three main approaches to obtain quantitative data on cardiac motion. The first one is to use dedicated imaging modalities that directly measure motion information. Echocardiographic Doppler Tissue Imaging [1], Magnetic Resonance (MR) tagging [2] and several other phase encoding MR sequences [3] have been developed to directly measure displacement or velocity fields for cardiac motion analysis [4], [5], [6]. However, those modalities are not widely used in clinics and give access to limited components of these fields (often one or two dimensional). Even with tridimensional tagging [7], it remains challenging to extract accurately, and to evaluate the quality of the recovered motion from another imaging modality [6] as it requires intermodality temporal and spatial registration.

A second one is to image a physical phantom with known shape and controlled motion [8], [9]. However, those phantoms are costly to design and manufacture and often lead to images with a limited realism compared to clinical images. This is due to the lack of the surrounding structures, and the difficulty to reproduce the complex cardiac motion.

The third method is to completely model the physics of the image acquisition system and of the organ of interest, and compute a numerical simulation of the resulting image. This was applied in the context of MR brain images [10], [11], cardiac MR sequences [12], tridimensional echocardiographic (US) sequences [13], [14], [4], [15] and Computed Tomographic (CT) images [16], [17]. A platform was recently developed to facilitate the access to several medical image simulators [18]. This approach has proved to be useful for many validation tasks. For instance, synthetic cardiac SPECT image sequences [19] were created for the validation of the developed 4D cardiac image segmentation [20]. Synthetic 2D ultrasound sequences [21] were also generated for the validation of a non-rigid registration method [22]. Finally, in order to validate a $3 \mathrm{D}$ strain estimation method of ultrasound images [4], an adapted convolutional model [23] was used to simulate US data sets. However, these approaches are computationally expensive and are restricted to a number of objects in the simulated field of view due to the complexity of the physical models involved. For cardiac sequence simulations, these constraints often impose to only simulate an isolated myocardium without much consideration of the blood pool and surrounding structures even though recently more complex models have been developed [24], [25]. Therefore it limits the realism of the created synthetic images. 


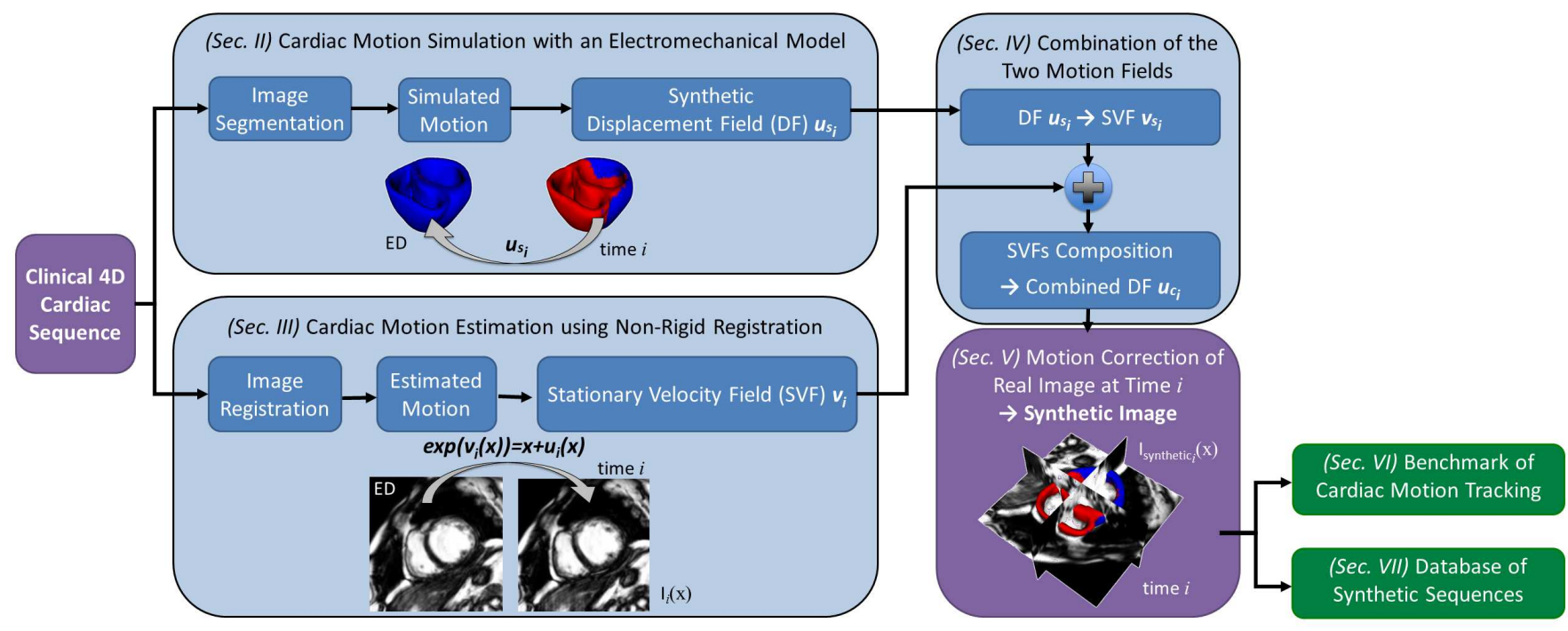

Fig. 1. Synthetic 4D Cardiac Sequence Generation Pipeline. A clinical 4D sequence is used as an input to create a synthetic 4D sequence in which the myocardium motion follows a prescribed simulated displacement field. A cardiac E/M model simulates the cardiac contraction using a cardiac mesh created from the myocardium segmentation at ED. Log Demons registration method is applied to all images in the real clinical sequence to register them to the ED reference image. The combined simulated and registration motion are used to deform the real clinical images in order to create the synthetic cardiac sequence. Two applications are presented: non-rigid registration benchmark and image database generation.

In this paper, we propose a new approach for creating synthetic and visually realistic cardiac image sequences based on real clinical image sequences, an electromechanical (E/M) model of the heart and an image registration algorithm. The method described below bypasses the simulation of the physics of acquisition by warping an original image sequence and replacing the observed motion of the myocardium by a simulated one. Without mimicking the physics of the acquisition, it uses the existing information in the real sequence to create the synthetic one. A key component to achieve this, is an E/M model of the heart. We use here a simple model [26] and an improved one [27], [28], but the methodology would remain the same with other models (see for instance [29], [30], [31] and references therein). After the geometry and the biophysical parameters are personalised [32], [33], [34], [35], [36], such a model can beat similarly to the apparent cardiac motion in a given sequence. Therefore we can have a simulated motion that is close to the one observed in the original image. The output of the proposed approach is a synthetic image sequence including the myocardium and its close environment (blood pools, valves...) that looks similar to the original sequence but where the myocardial motion is modified in a controlled manner and results from a simulation with known biophysical parameters. The motion of the structures surrounding the myocardium is slightly modified to follow the simulated myocardium motion.

Compared to the previous methods, the proposed approach leads to images that remain very similar to the original clinical images and where the cardiac motion is known everywhere. It does not require complex physical simulations of the acquisition system and may be applied to various modalities as shown in this paper. Synthetic images of brain atrophy were simulated using MR images and generated displacement fields in [37] but without taking into account real longitudinal sequences, only from a single time-point. In [38], simulated sequence is created by using a kinematic model to deform a real taggedMR image at end diastolic time point. We propose here to use a full time sequence of real data and a biophysical model of the heart, which are two very important points to obtain realistic sequences with large deformations. Using the whole real sequence information would also give better realism in the texture of the created sequence. It will also contain the changes of the surrounding environment such as the motion of the mitral valve.

The overall approach is described in Fig. 1 where the simulation of cardiac motion, the clinical cardiac motion estimation and the generation of synthetic sequences are respectively described in Sec. II, Sec. III and Sec. IV. In Sec. V, examples of synthetic sequences for cine MRI, 4D-CT and 4D-US imaging modalities are provided with an evaluation of their realism. Then, two applications are presented. The first one (Sec. VI) is the evaluation of a cardiac motion tracking algorithm (iLogDemons). The quantitative analysis of the synthetic images with a non-rigid registration algorithm allows to compare the estimated motion with the ground truth motion. The second application is the creation of a database of synthetic images (Sec. VII). It illustrates the application of this framework to help in developing image analysis methods based on machine learning [39].

\section{Cardiac Motion Simulation Using an Electromechanical Model of the Heart}

The first step in the proposed pipeline consists in simulating a cardiac motion. For this purpose, we used the cardiac E/M model proposed in [26]. We personalised the anatomy of the heart with the myocardium segmentation of a real clinical image at a reference time (end diastole / ED). The segmentation was done using an interactive 3D segmentation tool available within the CardioViz3D software [40] (cf. Fig. 2). Using 


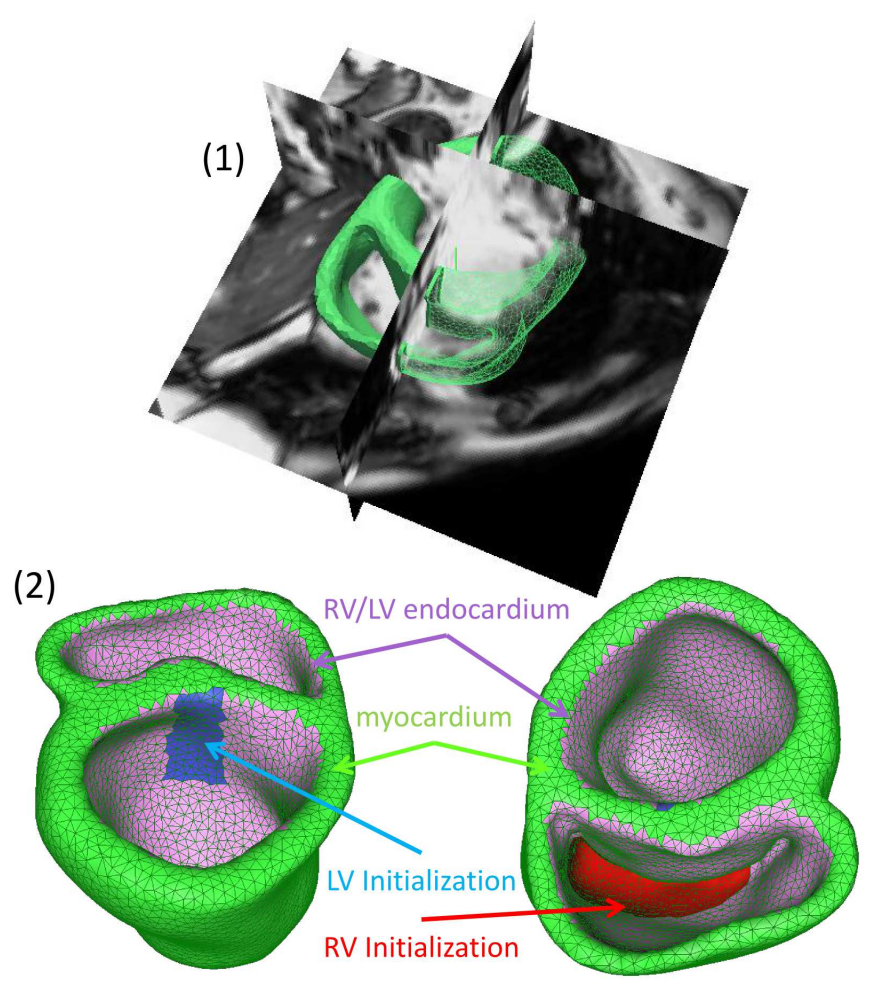

Fig. 2. Cardiac Segmentation, Mesh Geometry and Electrophysiological Initialization. (1) Myocardium segmentation is obtained by using an interactive segmentation tool within the CardioViz3D software. (2) Initial electrical activation area is set for the LV (blue) and RV (red). RV/LV endocardium (purple) is set to have higher conductivity $v$ than the rest of the myocardium (green) to represent the Purkinje network.

this tool, the 3D left ventricle (LV) and right ventricle (RV) endocardium and epicardium surfaces were segmented. Then a binary mask of the compact biventricular myocardium was created. A computational tetrahedral mesh which is suitable for the simulation was then generated from this myocardium mask using CGAL [41] available within the iso2Mesh tool proposed by [42].

From a personalised anatomy, we generated an electrophysiological activation pattern using the dynamic multifront Eikonal model which calculates the depolarization time $T_{d}$ at each point of the mesh [43]. The Eikonal equation $v \sqrt{\nabla T_{d}^{t} D \nabla T_{d}}=1$ was solved using the multi-front Fast Marching Method which also calculates the repolarization time $T_{r}$. In this equation, $v$ is the local conduction velocity and $D=(1-r) f \otimes f+r . I$ is the anisotropic conductivity tensor in Cartesian coordinates as defined in [26] where $f$ is the fibre orientation and $r$ is the conductivity anisotropy ratio. Synthetic myocardial fibres were created by varying the elevation angle (w.r.t. the short axis plane) of the fibre from $-80^{\circ}$ on the epicardium to $0^{\circ}$ at mid-wall to $+80^{\circ}$ on the endocardium. The initial electrical activation area was defined on the previously created cardiac mesh (cf. Fig. 2). We defined some areas in the LV and the RV which prescribe the His bundle proximity where the electrical propagation wave starts. We also set the LV and RV endocardium surfaces to have higher conductivity (larger $v$ ) compared to the rest of the myocardium, to represent the Purkinje network.
The obtained $T_{d}$ and $T_{r}$ were then used in the electromechanical coupling written as

$$
\begin{cases}\text { if } T_{d} \leq t \leq T_{r} & : \sigma_{c}(t)=\sigma_{0}\left(1-e^{\left(\alpha_{c}\left(T_{d}-t\right)\right)}\right) \\ \text { if } T_{r}<t<T_{d}+H P & : \sigma_{c}(t)=\sigma_{c}\left(T_{r}\right) e^{\left(\alpha_{r}\left(T_{r}-t\right)\right)}\end{cases}
$$

which gives the stress tensor $\sigma_{c}$ at time t. $H P$ is the heart period, $\alpha_{c}$ is the contraction rate, and $\alpha_{r}$ is the relaxation rate. The force vector $F_{c}$ was obtained from $F_{c}=\int_{S}\left(\sigma_{c} f \otimes f\right) n d S$ with $n$ the surface normal and $S$ the element surface. The dynamic equation which governs the displacement vector $U$ was written as $M \ddot{U}+C \dot{U}+K U=F_{b}+F_{c}$ where $M, C$ and $K$ are the mass, damping, and stiffness matrices respectively. $F_{b}$ is the external load from the boundary condition.

We also used an improved E/M model, Bestel-ClémentSorine (BCS) [28], which includes a passive non linear elastic part and an active part that describes more accurately the binding and unbinding processes of the actin and myosin filaments in the sarcomere. It also allows the Starling effect by which the maximum contraction depends on the fibre strain and includes the dissipation due to friction during the contraction. As a result, the simulated deformation incorporates twisting motion which makes it more realistic. This model was shown to provide realistic simulation of $\mathrm{LBBB}$ and heart failure, as well as accurate prediction of ventricular pressure changes with resynchronisation therapy [27].

For all the parameters of the electromechanical model we took into account the known condition of the heart in the real image we used. We could for instance include a Left Bundle Branch Block (LBBB) or a reduced contractility in an infarcted area. For the $\mathrm{LBBB}$ case, a detailed personalisation of the electrophysiology and mechanics models on two patients [27] has allowed us to characterise the parameter range associated with this pathology. From this experience, LBBB is simulated using a lower electrical conduction velocity $(\mathrm{v}=30 \mathrm{~cm} / \mathrm{s})$ and a reduced contractility $\left(\sigma_{0}=50\right)$ compared to the simulation of the normal case with $\mathrm{v}=50 \mathrm{~cm} / \mathrm{s}$ and $\sigma_{0}=90$. The Left Ventricle (LV) initialization (cf. Fig. 2) is also blocked to simulate LBBB. This simple personalisation of LBBB leads to reasonable simulated motion which is not too far from the observed one.

We also used a calibration method to assess the mechanical parameters of the BCS model so that the simulation is close to the real clinical sequence in term of ejected blood volume. We used the algorithm derived from the Unscented Transform [44], and described in [45]. The algorithm finds a set of parameters that enable the simulation to match observations on the endocardial volume of the LV (the minimum volume, the minimum and maximum of the flow) of the real clinical sequence in one iteration through the analysis of the covariance matrix between the simulated observations and the variation of each parameter independently.

This simulation provides a dense motion field within the myocardium for the whole cardiac cycle. This motion field can be sampled at the resolution of the original image sequence both in space and time. 


\begin{tabular}{ll}
\hline DF & displacement field \\
SVF & stationary velocity field \\
ED & end diastolic \\
$I_{i}(\mathbf{x})$ & real clinical image at time $i$ \\
$I_{\text {synthetic }}(\mathbf{x})$ & generated synthetic image at time $i$ \\
$\mathbf{u}_{\mathbf{s}_{i}}(\mathbf{x})$ & simulated DF at time $i$ \\
$\mathbf{v}_{\mathbf{s}_{i}}(\mathbf{x})$ & simulated SVF at time $i$ \\
$\mathbf{u}_{i}(\mathbf{x})$ & DF obtained from the registration of $I_{i}(\mathbf{x})$ to the \\
& reference ED real image. \\
$\mathbf{v}_{i}(\mathbf{x})$ & SVF obtained from the registration of $I_{i}(\mathbf{x})$ to the \\
& reference ED real image. \\
$\mathbf{v}_{\mathbf{c}_{i}}(\mathbf{x})$ & combined SVF at time $i$ \\
$\mathbf{u}_{\mathbf{c}_{i}}(\mathbf{x})$ & combined DF at time $i$ \\
$\phi_{c_{i}}(\mathbf{x})$ & transformation which transform $I_{i}(\mathbf{x})$ to \\
& $I_{\text {synthetic }_{i}}(\mathbf{x}) . \phi_{c_{i}}(\mathbf{x})=\mathbf{x}+\mathbf{u}_{\mathbf{c}_{i}}(\mathbf{x})$ \\
\hline & \multicolumn{2}{c}{} \\
\hline
\end{tabular}

TABLE I

NOTATIONS. DEFINITIONS OF THE NOTATION USED IN THE COMBINATION OF SIMULATED AND ESTIMATED MOTION

\section{CARDiaC MOtion ESTIMATION FROM IMAGES USING NON-RIGID REGISTRATION}

To be able to introduce a given cardiac motion within a clinical 4D image sequence, one has first to estimate the motion visible in this image, in order to replace it. To achieve this, we applied a non-rigid registration algorithm to the clinical $4 \mathrm{D}$ cardiac sequence to find the deformation field between each pair of images. We used the symmetric Log-Domain Demons (LogDemons) non-linear registration algorithm proposed in [46]. The purpose of applying this nonlinear image registration is to find the displacement vector field $\mathbf{u}(\mathbf{x})$ associated with the transformation $\phi(\mathbf{x})=\mathbf{x}+\mathbf{u}(\mathbf{x})$ which aligns a template image $\mathrm{T}(\mathbf{x})$ to a reference image $\mathrm{R}(\mathbf{x})$, where $\mathbf{x} \in \mathbb{R}^{3}$ is the space coordinate $(\operatorname{voxel}(x, y, z))$.

Here we took the end diastolic (ED) image as the reference image $\mathrm{R}(\mathbf{x})$. With this non-rigid registration algorithm, the displacement field $\mathbf{u}_{i}(\mathbf{x})$ between the ED image and each image at frame $i$ of the clinical 4D cardiac sequence was estimated (cf. Fig. 1). An accurate registration transforms each image at frame $i$ in the clinical 4D sequence well aligned to the ED reference image. Transformation $\phi_{i}(\mathbf{x})$ is parameterized by a stationary velocity field $\mathbf{v}_{i}(\mathbf{x})$ through the exponential map $\phi_{i}(\mathbf{x})=\exp \left(\mathbf{v}_{i}(\mathbf{x})\right)=\mathbf{x}+\mathbf{u}_{i}(\mathbf{x})$. Using this parameterization, we ensure the one-to-one correspondence between all points in the transformation since this is a physical property of the cardiac deformation (no tearing or destruction of tissue). With this method, the clinical image sequence was "stabilised" or "freezed", meaning that the apparent cardiac motion was removed from the sequence. We can then introduce the groundtruth motion generated by the biophysical model. We use the implementation of LogDemons algorithm in ITK [47]. For the registration parameters, 1 voxel size of regularization kernel and 3 or 5 multi-resolution levels (depending on the resolution of the image) with 200 iterations for each level usually gives good result.

\section{Combination of Simulated and Estimated Motions to Create Synthetic SEquences}

To combine simulated and estimated motions, we first adjusted temporally the simulated motion and then sampled it in

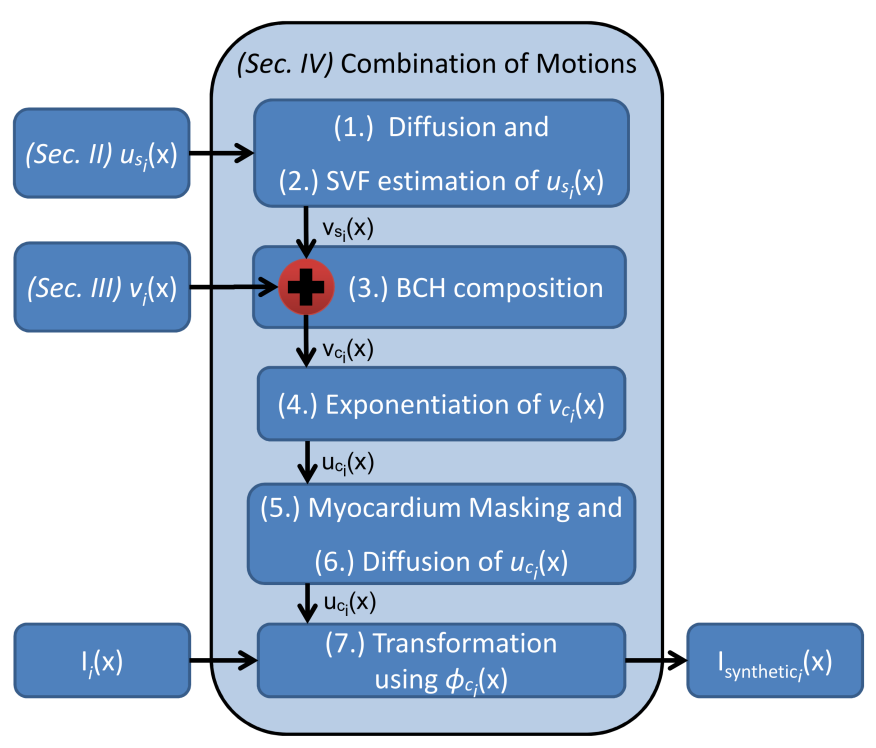

Fig. 3. Block Diagram of the Motion Combination Process. The simulated and the estimated motions are combined to transform the real image.

order to match the cycle length and temporal resolution of the clinical 4D sequence. The sampling of the simulated motion consists in computing the displacement field (DF) between the reference configuration (ED) and the deformed position at each time frame of the sequence. This displacement is only known at the vertices of the tetrahedral meshes, but using the linear interpolation inside each tetrahedron, we rasterized the continuous DF into a volumetric image having the same size and spatial resolution as the clinical 4D sequence.

The steps are summarized in Fig 1. They include the warping of clinical image $I_{i}(\mathbf{x})$ at time $i$ into the synthetic image $I_{\text {synthetic }}(\mathbf{x})$. This warping mainly occurs around the myocardium, and the amount of deformation depends on how much the simulated cardiac motion differs from the one estimated in the original image sequence.

The transformation $\phi_{c_{i}}(\mathbf{x})$ of the original image is obtained through several computing stages. However, it can be simply formulated as resulting from the composition of the transformation from time $i$ to ED estimated through the Symmetric Log-Demons algorithm with the transformation from ED to time $i$ which was simulated by the electromechanical model of the heart.

The pipeline to create $\phi_{c_{i}}(\mathbf{x})$ is sketched in Fig. 1 and Fig. 3 and detailed in Alg. 1.

A first step consists of transforming the simulated DF $\mathbf{u}_{\mathbf{s}_{i}}(\mathbf{x})$ into a diffeomorphic transformation parameterized by a stationary velocity field $\mathbf{v}_{\mathbf{s}_{i}}(\mathbf{x})$. Indeed, it is important to handle smooth and diffeomorphic transformations to avoid any tearing or folding of the image textures. To achieve this, we extrapolated the simulated DF outside the myocardium using a diffusion method described in Alg. 2. The extrapolation is necessary to estimate the velocity field as it is sensitive to discontinuity of displacements. The diffusion of a vector field outside the myocardium is based on the iterative convolution of a Gaussian kernel followed by the reinitialisation of vector values in myocardium and border voxels. We used the recur- 

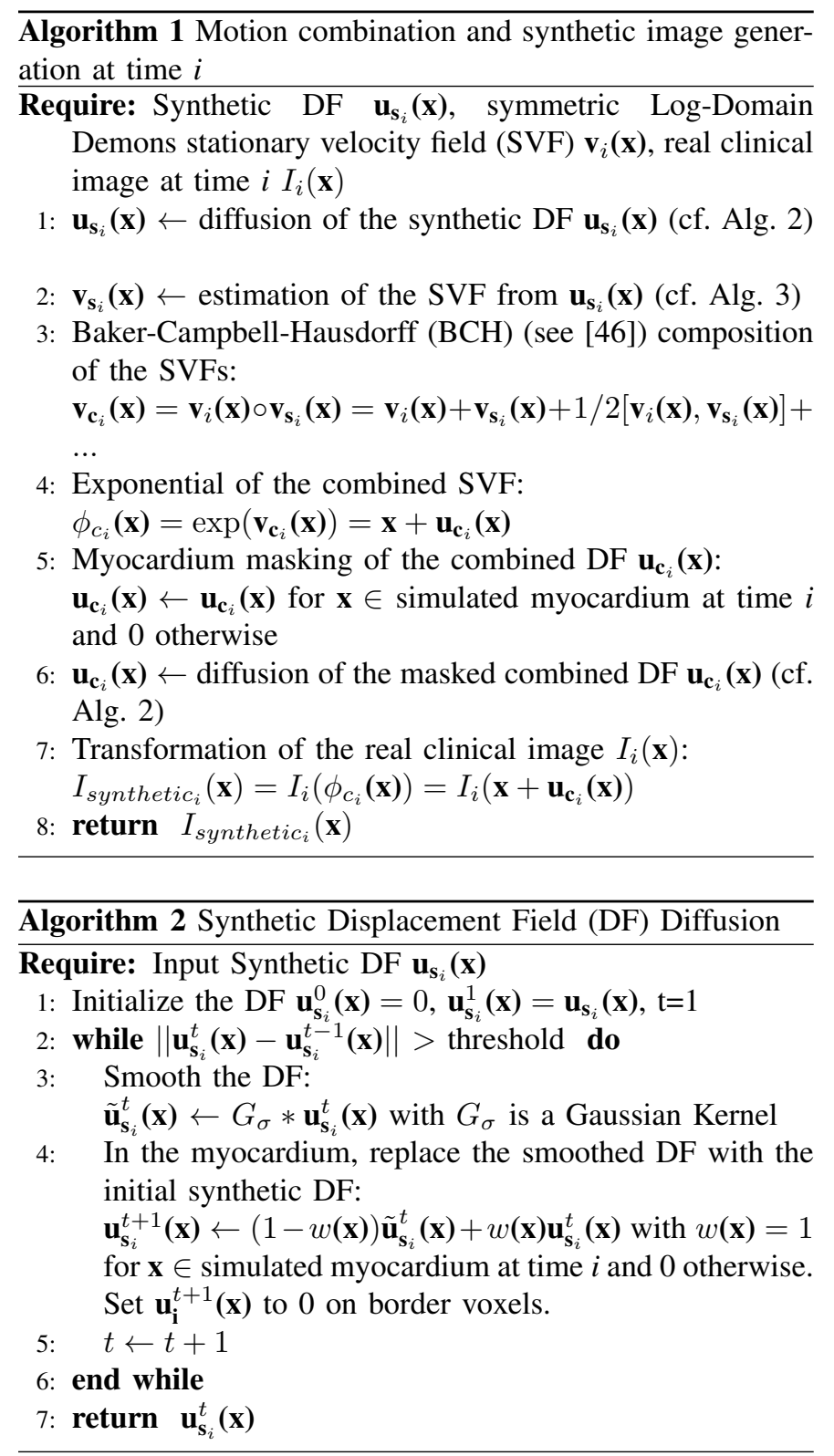

sive Gaussian filter implemented in ITK with 1 voxel size of sigma. The process is iterated until the difference in vectors between two iterations is less than $1 \%$ of the smallest voxel size.

We then used the algorithm proposed by [48] as implemented in [47] to estimate the synthetic stationary velocity field $\mathbf{v}_{\mathbf{s}_{i}}(\mathbf{x})$ from the synthetic DF $\mathbf{u}_{\mathbf{s}_{i}}(\mathbf{x})$, see Alg. 3. The estimation of the velocity field from the displacement field described in Alg. 3 requires the computation of the exponential of a stationary velocity field. We used here a forward Euler integration scheme as the more standard "Scaling and Squaring" method could have a limited accuracy in large deformation cases.

In a second step, the symmetric Log Demons registration and the extrapolated diffeomorphic simulated transformation are composed. Since the symmetric Log Demons algorithm also estimates a transformation parameterized by a stationary velocity field $\mathbf{v}_{i}(\mathbf{x})$, this composition can be done conveniently

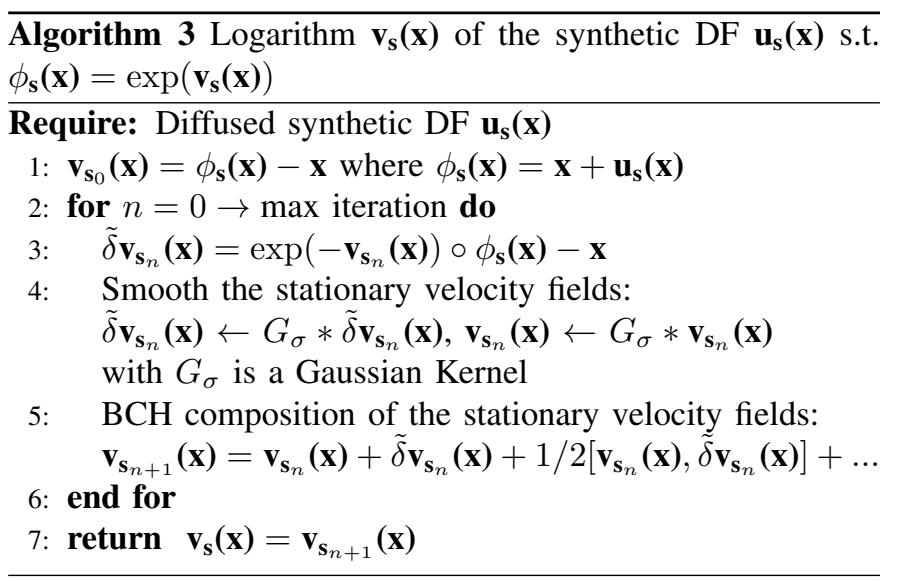

in the space of velocities using the Baker-Campbell-Hausdorff $(\mathrm{BCH})$ rule (see [46]) : $\mathbf{v}_{\mathbf{c}_{i}}(\mathbf{x})=\mathbf{v}_{i}(\mathbf{x}) \circ \mathbf{v}_{\mathbf{s}_{i}}(\mathbf{x})$. This composition is meaningful because velocity field $\mathbf{v}_{\mathbf{s}_{i}}(\mathbf{x})$ shows the positions at ED for each point in the simulated myocardium at time $i$ and the velocity field $\mathbf{v}_{i}(\mathbf{x})$ points to the position at time $i$ in the real clinical image for each point at ED. An important fact is that the simulated myocardium at ED is the same as the myocardium segmented in the ED clinical image (cf. Sec. II). Therefore we are filling the simulated myocardium at time $i$ with the myocardium intensity of real image at the same time.

In the last step, we corrected the displacement field $\mathbf{u}_{\mathbf{c}_{i}}(\mathbf{x})=$ $\exp \left(\mathbf{v}_{\mathbf{c}_{i}}(\mathbf{x})\right)-I d(\mathbf{x})$ outside the myocardium in order to keep most of the synthetic image similar to the real clinical image. To this end, we set to zero the DF outside the simulated myocardium by masking $\mathbf{u}_{\mathbf{c}_{i}}(\mathbf{x})$ with the simulated myocardium mask at time $i$. Then, we applied the same diffusion process as in Alg. 2 to smooth the field and enforce that structures surrounding the myocardium follow the motion of the myocardium. Finally the resulting displacement field is applied to the image $I_{i}(\mathbf{x})$ to create $I_{\text {synthetic }}(\mathbf{x})$.

\section{Producing Synthetic 4D Image Sequences}

The processes were performed on a desktop PC with Intel Xeon processor at $3.33 \mathrm{GHz}$. The computation time is a function of the resolution of the image. The LogDemons registration takes 1 hour for each pair of CT images and 5 minutes for MRI. For the cardiac motion simulation, we used the BCS E/M model which was implemented in the SOFA simulation platform [49]. It takes 20 minutes for a cardiac cycle simulation. Without code optimization, the next steps which include the motion combination up to the generation of the synthetic image take 3 hour for a synthetic CT image and 2 minutes for MRI."

\section{A. Synthetic Cine MRI Sequence}

We applied our proposed approach to create a synthetic cine MRI sequence from a clinical cine MRI sequence of an 84 years old male patient with dilated cardiomyopathy and left bundle branch block (LBBB) acquired on Achieva MR Philips Medical System scanner with the following acquisition protocol; Multi-slice steady state free precession (SSFP) sensitivity encoding (SENSE) with 30 views per gating interval, echo 


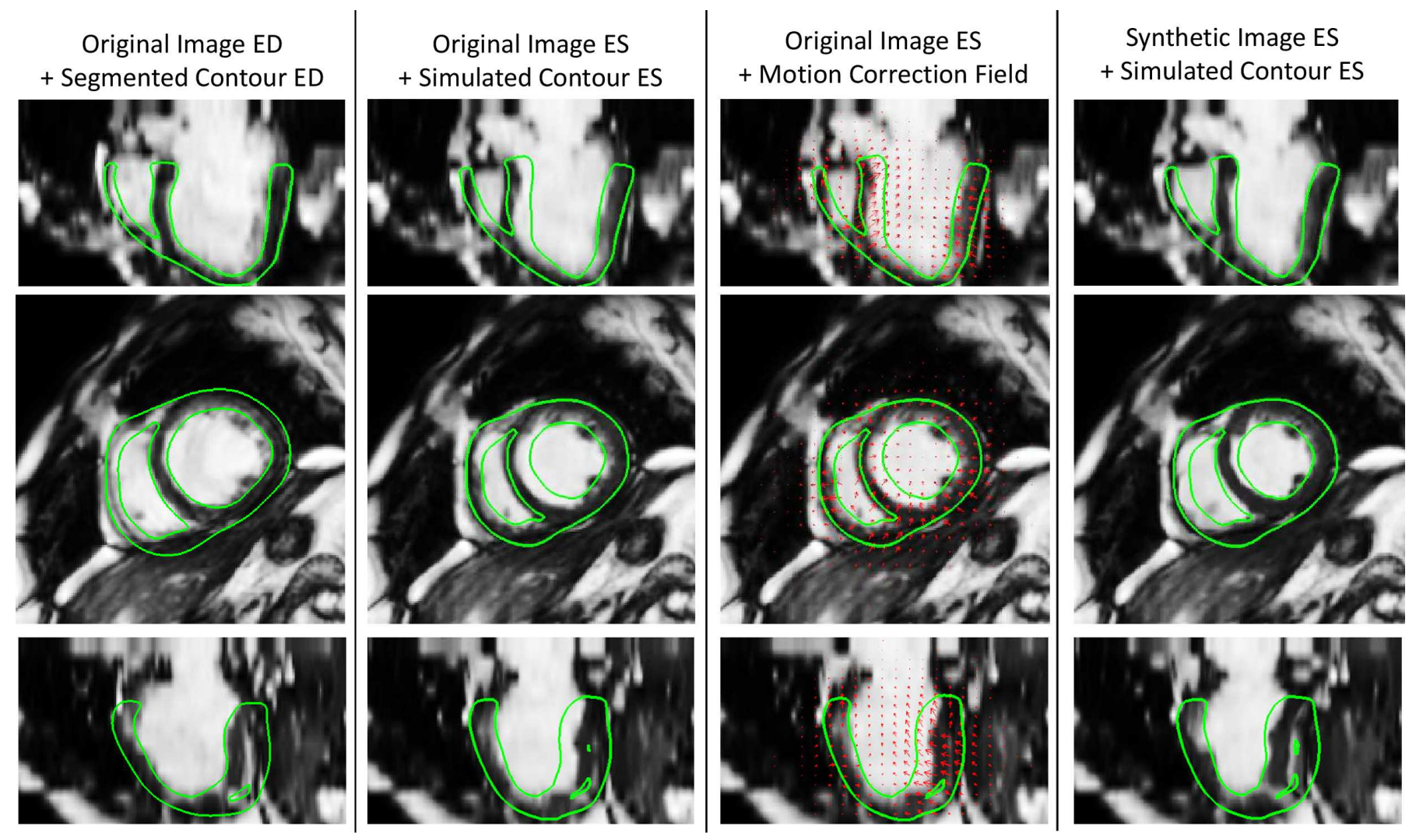

Fig. 4. Synchronized Synthetic cine MRI Created from Pathological Sequence. End diastolic clinical image is shown with its segmentation. The original clinical image at ES is shown with the contour of the simulated mesh at the corresponding time. The synthetic combined displacement field is applied to the original image at ES to correct the motion. The contour of the resulting synthetic image at ES shows a good agreement with the contour of the simulated mesh at ES. Video can be seen in the supplementary material.

time of $1.5071 \mathrm{~ms}$, repetition time of $3.0142 \mathrm{~ms}$ and flip angle of $60^{\circ}$. The image size was $171 \times 171 \times 98$ voxels with the resolution of $1.424 \times 1.424 \times 1.424 \mathrm{~mm}^{3}$ (the image was isotropically resampled from an image with an original slice thickness of $8 \mathrm{~mm}$ ). The number of images in the sequence was 30 .

In order to be more illustrative, we simulated the resynchronisation of this heart to create the synthetic cine MRI sequence. It allowed to have a correction field of a larger magnitude and therefore to better evaluate the impact on the original image (see Fig. 4).

The proposed sequence generation method produced a visually realistic synthetic sequence with a seamless fusion of the simulated myocardial motion and the neighbouring moving structures. The contraction of the created synthetic cine MRI sequence follows the motion of the E/M simulation which is shown by the myocardium contour which corresponds to the contour of the deformed mesh in the E/M simulation (cf. Fig. 4).

We also created a personalised synthetic sequence and compared the intensity histograms in the myocardium mask between the original and synthetic images. As expected, we observe that both intensity histograms are similar (cf. Fig. 7). It shows that the intensity distribution in the simulated myocardium is not too much distorted.

\section{B. Synthetic 4D CT Sequence}

A synthetic 4D CT sequence was created from a clinical 4D CT sequence obtained from a public clinical image database [50], acquired using a Siemens Sensation 64 CT system. Since the data is anonymized, no patient details are available. The ejection fraction of this patient is $61 \%$, therefore we believe this sequence presents a normal ventricular function. The image size was $512 \times 512 \times 377$ voxels with the resolution of $0.402 \times 0.402 \times 0.402 \mathrm{~mm}^{3}$ (resampled from the original slice thinkness of $2 \mathrm{~mm}$ ). The number of images in the sequence is 10 .

Again, in order to better demonstrate the method, we applied a simulation with a different condition than the original sequence. In this case, we created a left bundle branch block.

The contraction of the synthetic 4D CT also showed a good agreement with the motion of the E/M simulation, as demonstrated by the alignment of the contour of the myocardium with the mesh from the E/M simulation (cf. Fig. 5). From the created personalised simulation, the real and the synthetic CT image intensity histograms are very similar (cf. Fig. 7)

\section{Synthetic 4D US Sequences}

We used a clinical sequence acquired with a iE33 Philips Echocardiography System probe on a healthy 33 years old male volunteer. The image size was $224 \times 208 \times 208$ voxels with the resolution of $0.727 \times 0.716 \times 0.632 \mathrm{~mm}^{3}$. The iE33 


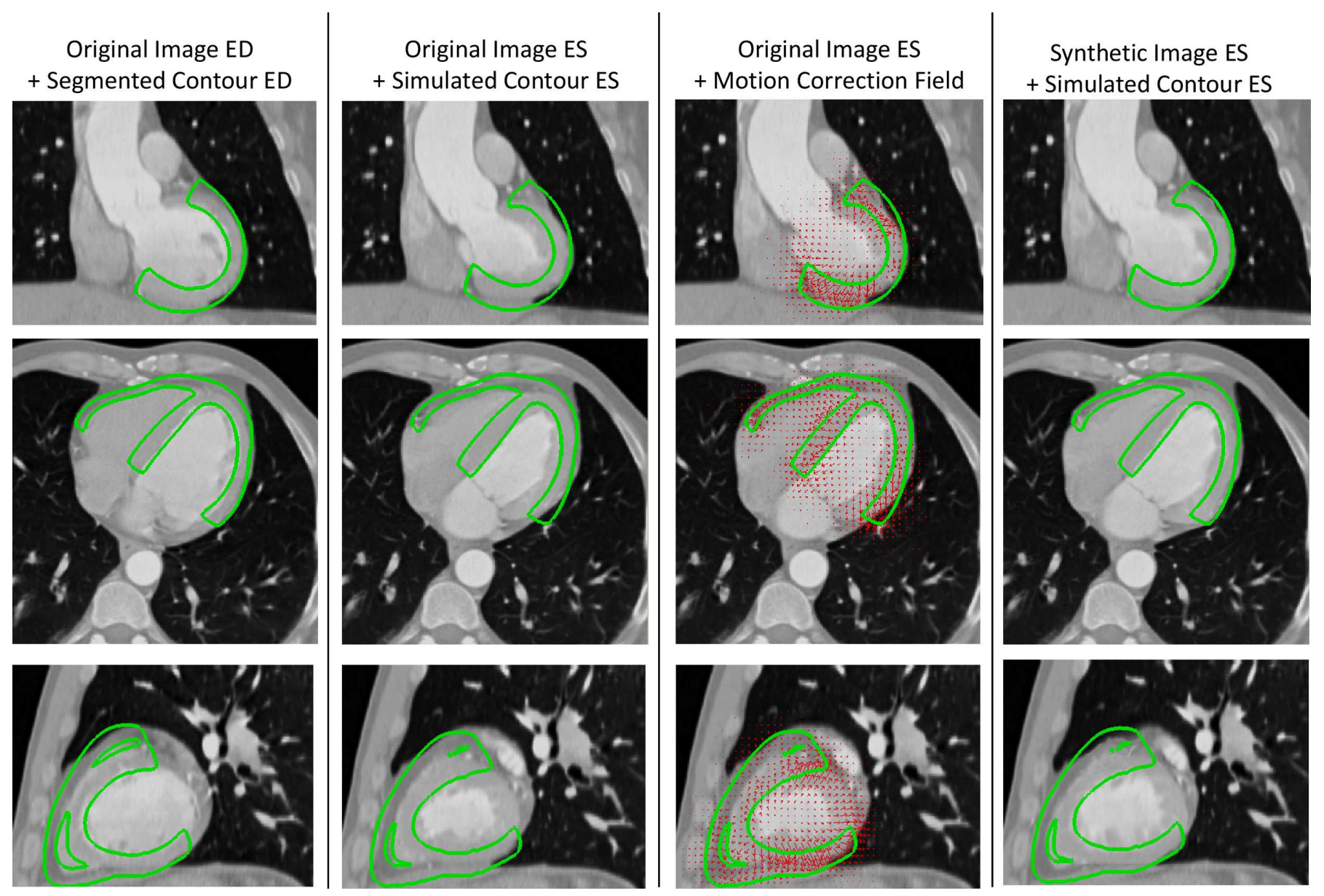

Fig. 5. Pathological Synthetic 4D CT Created from a Clinical Sequence. End diastolic clinical image is shown with its segmentation. The original clinical image at ES is shown with the contour of the simulated mesh at the corresponding time. The synthetic combined displacement field is applied to the original image at ES to correct the motion. The contour of the resulting synthetic image at ES shows a good agreement with the contour of the simulated mesh at ES. Video can be seen in the supplementary material.

system acquired a fully sampled cardiac volume within four cardiac cycles. The number of images in a cardiac cycle was 30 .

Generating synthetic US sequences was more challenging than other modalities because of the reduced field of view. The myocardium is not completely visible in the image, thus one has to manage the difference between the myocardial tissue entering the image during contraction in the clinical sequence and in the synthetic one. Additional information might be needed for outside of the ultrasound acquisition cone.

In order to tackle this, different steps were added. First, the field of view was artificially expanded by dilating the acquisition pyramid. We copied the boundary voxels with additional noise to fill this region. It provided an approximation of the image intensity for voxels entering the cone. Then after the synthetic image was generated, the image was cropped at the original size. Finally, the displacement field outside the 3D US acquisition cone was set to be zero during the last diffusion process of the masked displacement field as explained in Sec. IV.

We preserved the dynamics of the image, in particular the visible speckle pattern. We also preserved the realism of the ultrasound image quality gradient from base to apex in the synthetic image like in the clinical image where the apical quality is better than the basal quality. The contraction of the synthetic sequence follows the motion of the E/M simulation which was used to create the synthetic sequence. In Fig. 6, the contour of the cardiac mesh at end systole was overlaid on the synthetic 3D image at the same time point. The myocardium contour of the synthetic sequences correctly followed the contour of the deformed cardiac mesh from the E/M simulation.

We compared the intensity histograms of the original and the personalised synthetic images (cf. Fig. 7); they look similar and tend to follow a typical Rayleigh distribution which is a characteristic of US cardiac tissue images [23].

\section{Benchmark of Cardiac Motion Tracking}

As an illustrative application we performed cardiac motion tracking on synthetic sequences and compared the results with the ground-truth motion. Compared to the images shown in the previous section, we used here the simple personalised models so that the motion correction is reduced, and therefore the image texture minimally modified.

We tested here the iLogDemons algorithm [6]. Note that this method is different from the one which was used to generate 


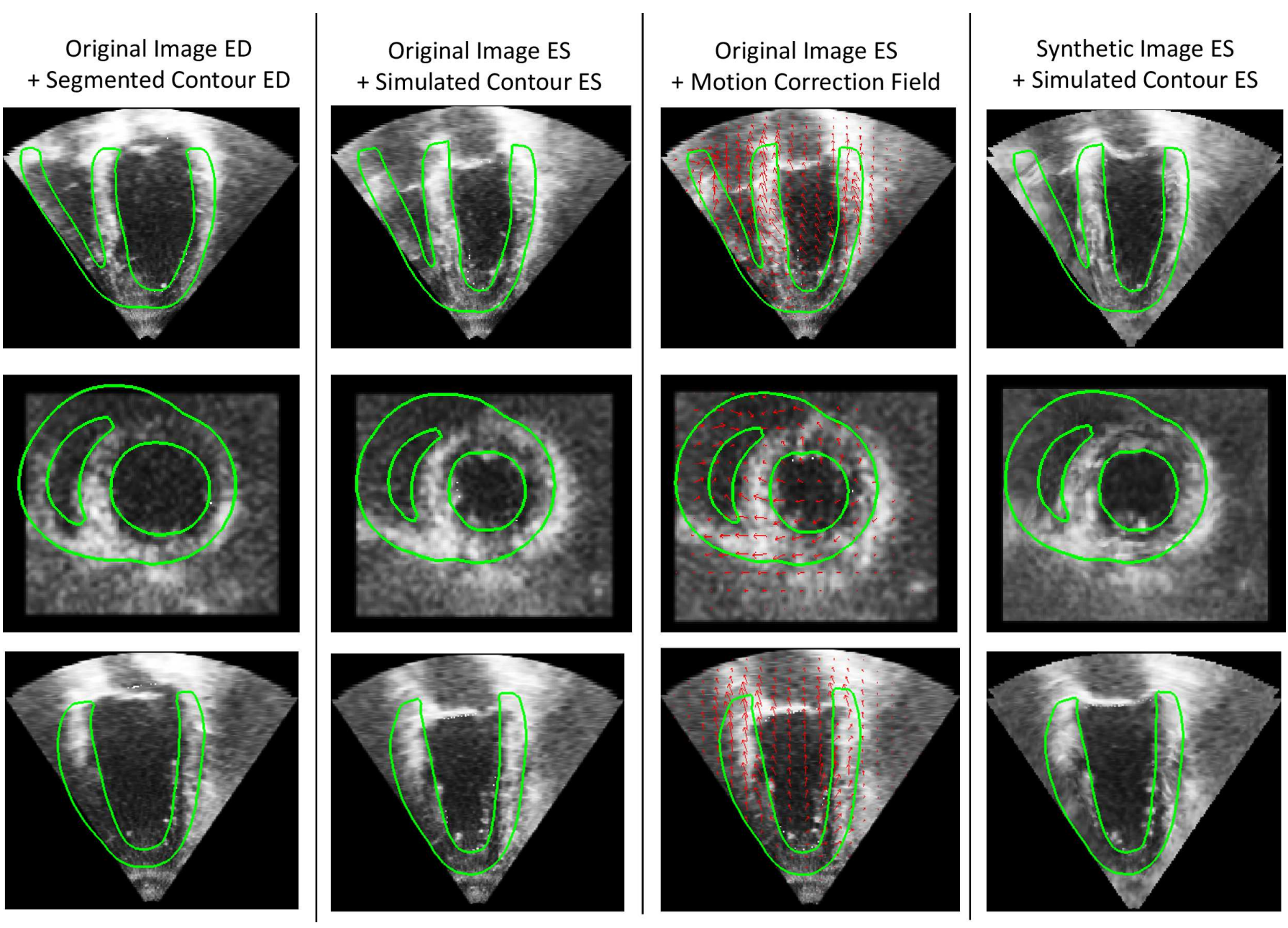

Fig. 6. Pathological Synthetic 4D US Created from Volunteer Data. End diastolic clinical image is shown with its segmentation. The original clinical image at ES is shown with the contour of the simulated mesh at the corresponding time. The synthetic combined displacement field is applied to the original image at ES to correct the motion. The contour of the resulting synthetic image at ES shows a good agreement with the contour of the simulated mesh at ES. Video can be seen in the supplementary material.
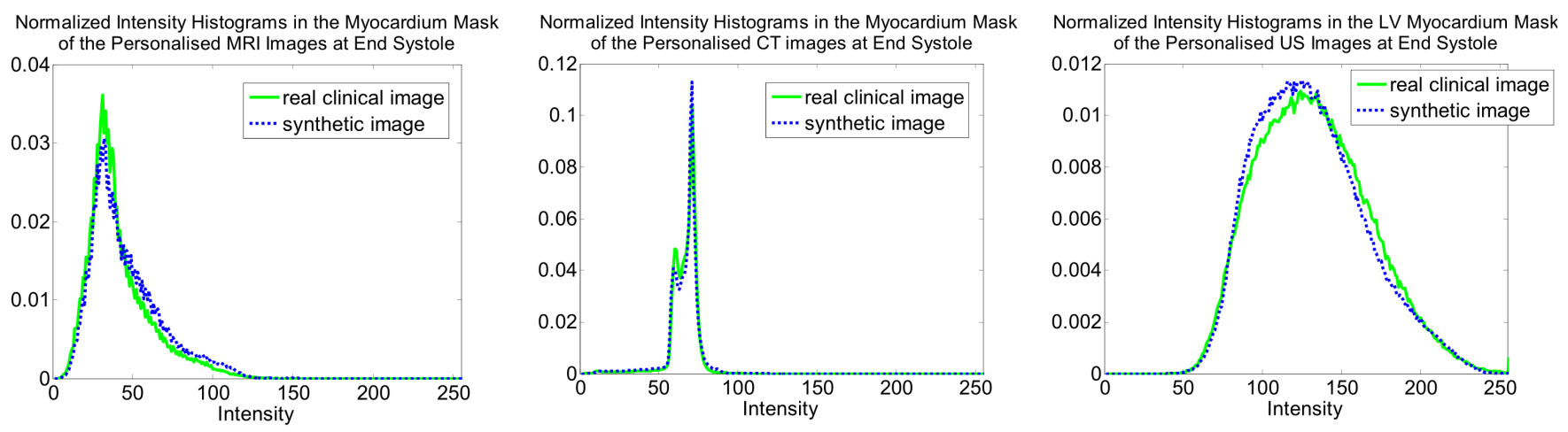

Fig. 7. Intensity Histograms Comparison. Similar intensity histograms are shown from the synthetic and the real clinical image at ES. 


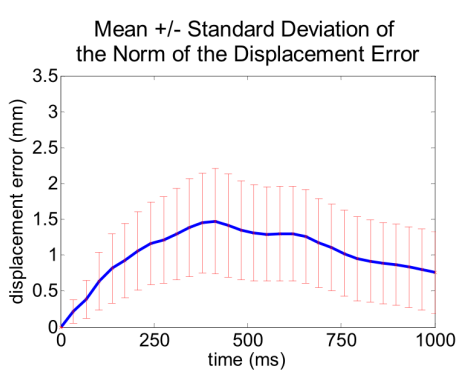

Pathological Synthetic cine MRI from Patient Sequence

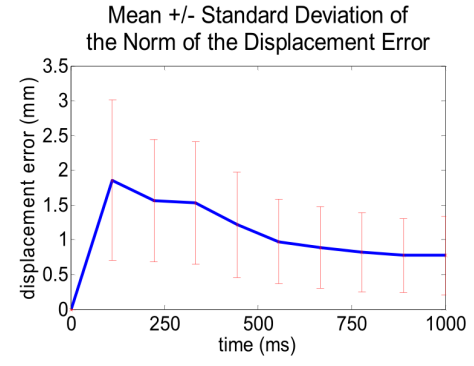

Normal Synthetic 4D CT from Patient Sequence

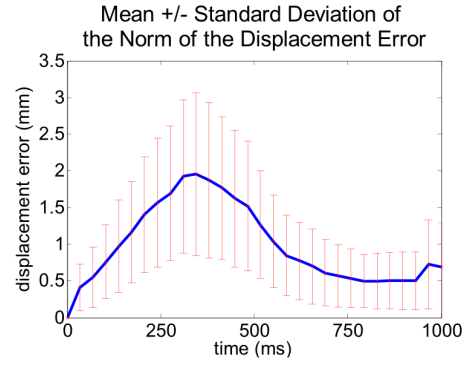

Normal Synthetic 4D US from Volunteer Sequence

Fig. 8. Ground Truth and iLogDemons Estimated Displacement Field Difference. From the synthetic sequences, the norm of the displacement field differences between the E/M simulated and the iLogDemons estimated motion was computed. The mean and standard deviation during the cardiac cycle are shown.

the images, as this method enforces the incompressibility of the myocardium during the cardiac motion tracking to regularize the visible motion in the image sequence and better recover the full motion. A binary mask from the ED segmentation is used to apply the incompressibility. The parameters of the tracking algorithm were taken as in [51], where a regularisation kernel of the size of the voxel was used.

The motion tracking estimates the DF $\mathbf{u}_{\mathbf{r}_{i}}(\mathbf{x})$ between each image at time $i$ in the synthetic sequence and the ED reference image. To evaluate the iLogDemons results, we computed the norm of the displacement difference between the ground truth and the iLogDemons motion fields: $\left\|\mathbf{u}_{\mathbf{s}_{i}}(\mathbf{x})^{-1}-\mathbf{u}_{\mathbf{r}_{i}}(\mathbf{x})\right\|$. We needed to inverse the ground truth motion that we used previously to generate the synthetic sequence (cf. Sec. IV) in order to compute $\mathbf{u}_{\mathbf{s}_{i}}(\mathbf{x})^{-1}$, but this is easily done as we directly estimate this motion field from meshes. The results are shown in Fig. 8. The maximum value of the mean displacement differences in the sequence are $1.48 \mathrm{~mm}, 1.86 \mathrm{~mm}$ and 1.95 $\mathrm{mm}$ for the MRI, CT, and US sequences respectively. While we can see that the iLogDemons achieves reasonable tracking results, it allows to quantify precisely the remaining error and therefore optimise the algorithm to improve the results.

\section{Database of Synthetic Sequences}

We illustrate here another application of this method: it is possible to multiply the number of images of a small database of typical clinical images, by creating similar but slightly different cases around each original sequence. To achieve this, we used a simple model personalised to a given sequence, and varied the parameters around the personalised values to generate additional cases.

The clinical sequences we used included a healthy volunteer and a heart failure patient, with a Left Bundle Branch Block (LBBB). Therefore different simulation scenarios were performed, including normal and pathological cases. In LBBB, the left ventricle (LV) initial electrical activation was blocked (cf. Fig. 2). The normal and heart failure cases have also different global contractility (adimensioned) mechanical parameters namely the peak contractility of the E/M coupling $\sigma_{0}$ previously discussed in Sec. II. The heart failure case peak contractility value is set to be lower than the normal case as failing hearts contract less. The global electrical conductivity

\begin{tabular}{lll}
\hline Original Image & Simulated Scenario & Clinical Decision \\
\hline Healthy sequence & Healthy model & Healthy heart \\
Pathological sequence & Pathological model & Pathological heart \\
Healthy sequence & Pathological model & Pathological heart \\
Failing heart sequence & Normal activation pattern & Resynchronised heart \\
\hline \multicolumn{2}{c}{ TABLE II } &
\end{tabular}

Clinical Evaluation. Clinical diagnosis done on Synthetic SEQUENCES GENERATED FROM THE GIVEN ORIGINAL CLINICAL IMAGES AND SIMULATED SCENARIOS.

parameter $v$ (cf. Sec. II), which is the conduction velocity $(\mathrm{cm} / \mathrm{s})$ of the electrophysiological model, is also set smaller in this failing heart.

We thus created a synthetic 4D US database containing the previous scenarios. Such database is useful for CRT where the $4 \mathrm{D}$ cardiac US is widely used to select the patients and evaluate the treatment. The normal simulation is used to create a healthy synthetic cardiac sequence, with controlled healthy electrical propagation and motion from the E/M simulation, using the volunteer clinical sequence. The LBBB simulation is used to create a pathological synthetic sequence from a pathological clinical sequence.

In order to test the limits of our approach, we also created a heart failure cardiac sequence with LBBB (cf. Fig. 6) from the clinical healthy volunteer sequence and a sequence with normal activation from the failing heart clinical image. A cardiologist performed a diagnostic evaluation on the generated synthetic US sequences (cf. Table. II). The simulated conditions were correctly diagnosed in the four cases, the normally activated failing heart being interpreted as a resynchronised heart, which is expected to have the anatomy of a failing heart but a synchronous motion.

Such databases can be used to develop machine-learning approaches as was shown in [52], [53] with a preliminary version of the approach described in this article.

\section{DISCUSSION}

In this study, we used the information from an existing real image sequence and deformed it using a known displacement field in order to create a synthetic new one. As a result, both of 
them are similarly looking. However, possible artefacts might occur in the synthetic sequence due to several reason.

First, if the deformation of the original real clinical sequence is too large, the motion tracking may fail to entirely stabilize the clinical data. Therefore the final motion still contains part of the original motion. As explained in Sec. IV, the estimated displacement field of the original sequence is used to construct the combined known displacement field. The variability in clinical image quality could also affect this motion tracking process since LogDemons registration method is based on the intensity of the image.

Second, if the simulated motion is too far from the patient motion, this large difference can warp the texture in an unrealistic way, as the original image has to be resampled with roughly the difference between the two motion fields. This is why the database has to be generated by varying the $\mathrm{E} / \mathrm{M}$ parameters around the original patient motion.

Third, the resulting synthetic image will have similar quality to the real one. For the MRI case, since we resampled our MR images to be isotropic, the resulting synthetic sequence has similar smooth edge transition in the long axis direction as the real sequence.

Finally, the generation of synthetic 4D US sequences requires delicate interpolations between Polar and Cartesian coordinates, because the registration process is performed in the Cartesian coordinates, as well as the computation of the next steps. Ideally the process should be done in Polar coordinates and only the final data should be re-interpolated in Cartesian coordinates in order to respect the effect of the acquisition on the texture.

Another limitation is related to the heart shape which is related to the original image sequence. Therefore the applied motion must correspond to the condition of the observed heart to be realistic. For instance, if the original sequence is from a patient with a dilated cardiomyopathy, this will appear in the synthetic images, even if we use electromechanical parameters corresponding to a healthy heart. It is better to simulate a closer motion to the original sequence. However, this can still allow the production of a resynchronized heart sequence before remodelling for instance. This could serve in the therapy planning.

Concerning the motion at the heart boundary, continuity is enforced by the diffusion of the motion inside the myocardium. The formulation minimizes the gradient of displacement field in order to avoid deforming as much as possible the texture. Better boundary conditions next to surrounding structures could be used to improve the motion profile in these areas, for instance through a pericardium model. The atria are considered as the surrounding environment since the E/M model does not include them. At the frontiers of the atria, continuity is preserved using the displacement field diffusion method.

The registration algorithm will be integrated in the medInria software and the cardiac simulation will be available in the SOFA platform. They are available at http://team.inria.fr/asclepios/software/.

\section{COnClusion}

We developed a pipeline to create visually realistic synthetic 4D cardiac sequences using the deformation from an electromechanical model simulation. This pipeline combines the simulated myocardium displacement with the estimated myocardium displacement from the original clinical images. This combined displacement field is then used to warp the original images in order to create the synthetic cardiac sequence.

In this pipeline, we proposed a new approach based on Stationary Velocity Fields to combine the motions. We also proposed a new method of motion diffusion in order to maintain the continuity of the simulation and the real image with minimal texture distortion. Thanks to the detailed interplay between image processing and biophysical modeling, we can fully use a complete sequence in order to generate several new ones. This method also gives better realism compared to the traditional method, namely deforming an end-diastolic image, since the generated synthetic sequence will also contain the changes of the surrounding environment such as the motion of the mitral valve.

The new synthetic images are similar to the original ones except for the motion of the heart which is modified to follow the motion provided by a biophysical model. The parameters of the biophysical model can be modified to create variations around this motion. This pipeline has been tested to generate different synthetic sequences from different imaging modalities. It is generic and can be used with a different biophysical model or a different image registration algorithm, and it can be extended to other organs.

As these synthetic 4D cardiac sequences have kinematic ground truth information, those sequences represent in themselves a valuable resource to benchmark motion tracking methods or to train machine-learning algorithms.

\section{ACKNOWLEDGEMENT}

This work was partially supported by the European Research Council through the ERC Advanced Grant MedYMA on Biophysical Modelling and Analysis of Dynamic Medical Images and the European project euHeart.

\section{REFERENCES}

[1] C. Y. Ho and S. D. Solomon, "A clinician's guide to tissue Doppler imaging." Circulation, vol. 113, no. 10, pp. e396-8, March 2006.

[2] X. Liu and J. Prince, "Shortest path refinement for motion estimation from tagged MR images," Medical Imaging, IEEE Transactions on, vol. 29, no. 8, pp. $1560-1572$, August 2010.

[3] C. Ozturk, J. Derbyshire, and E. McVeigh, "Estimating motion from MRI data," Proceedings of the IEEE, vol. 91, no. 10, pp. 1627 - 1648, October 2003.

[4] A. Elen, H. F. Choi, D. Loeckx, H. Gao, P. Claus, P. Suetens, F. Maes, and J. D'hooge, “Three-dimensional cardiac strain estimation using spatio-temporal elastic registration of ultrasound images: A feasibility study," Medical Imaging, IEEE Transactions on, vol. 27, pp. 1580-1591, 2008.

[5] M. D. Craene, G. Piella, N. Duchateau, E. Silva, A. Doltra, H. Gao, J. D'hooge, O. Camara, J. Brugada, and M. Sitges, "Temporal diffeomorphic free-form deformation for strain quantification in 3D-US images," in Medical Image Computing and Computer-Assisted Intervention - MICCAI 2010, ser. LNCS, T. Jiang, N. Navab, J. Pluim, and M. Viergever, Eds., vol. 6362. Springer Heidelberg, 2010, pp. 1-8. 
[6] T. Mansi, X. Pennec, M. Sermesant, H. Delingette, and N. Ayache, "iLogDemons: A demons-based registration algorithm for tracking incompressible elastic biological tissues," International Journal of Computer Vision, vol. 92, pp. 92-111, 2011.

[7] A. K. Rutz, S. Ryf, S. Plein, P. Boesiger, and S. Kozerke, "Accelerated whole-heart 3D CSPAMM for myocardial motion quantification," Magnetic Resonance in Medicine, vol. 59, no. 4, pp. 755-63, 2008.

[8] J.-F. Daisne, M. Sibomana, A. Bol, G. Cosnard, M. Lonneux, and V. Grégoire, "Evaluation of a multimodality image (CT, MRI and PET) coregistration procedure on phantom and head and neck cancer patients: accuracy, reproducibility and consistency," Radiotherapy and Oncology, vol. 69, no. 3, pp. $237-245,2003$.

[9] T. Boltz, W. Pavlicek, R. Paden, M. Renno, A. Jensen, and M. Akay, "An anthropomorphic beating heart phantom for cardiac X-ray CT imaging evaluation," Journal of Applied Clinical Medical Physics, vol. 11, no. 1, 2010.

[10] H. Benoit-Cattin, G. Collewet, B. Belaroussi, H. Saint-Jalmes, and C. Odet, "The SIMRI project: a versatile and interactive MRI simulator," Journal of Magnetic Resonance, vol. 173, pp. 97 - 115, 2005.

[11] B. Aubert-Broche, A. C. Evans, and L. Collins, "A new improved version of the realistic digital brain phantom," Neurolmage, vol. 32, pp. 138 $145,2006$.

[12] C. Tobon-Gomez, F. M. Sukno, B. H. Bijnens, M. Huguet, and A. F. Frangi, "Realistic simulation of cardiac magnetic resonance studies modeling anatomical variability, trabeculae, and papillary muscles," Magnetic Resonance in Medicine, vol. 65, no. 1, pp. 280-288, 2011.

[13] Q. Duan, P. Moireau, E. D. Angelini, D. Chapelle, and A. F. Laine, "Simulation of 3D ultrasound with a realistic electro-mechanical model of the heart," in Functional Imaging and Modeling of the Heart, ser LNCS, F. B. Sachse and G. Seemann, Eds., vol. 4466. Springer Heidelberg, 2007, pp. 463-473

[14] C. Butakoff, S. Balocco, S. Ordas, and A. F. Frangi, "Simulated 3D ultrasound LV cardiac images for active shape model training," in Medical Imaging 2007: Image Processing, ser. SPIE, J. P. W. Pluim and J. M. Reinhardt, Eds., vol. 6512, 2007, p. 65123U.

[15] O. Kutter, R. Shams, and N. Navab, "Visualization and GPU-accelerated simulation of medical ultrasound from CT images," Computer Methods and Programs in Biomedicine, vol. 94, pp. 250-266, June 2009.

[16] W. P. Segars, M. Mahesh, T. J. Beck, E. C. Frey, and B. M. W. Tsui, "Realistic CT simulation using the 4D XCAT phantom," Medical Physics, vol. 35, no. 8, pp. 3800-3808, 2008.

[17] A. Veress, W. Segars, B. Tsui, and G. Gullberg, "Incorporation of a left ventricle finite element model defining infarction into the XCAT imaging phantom," Medical Imaging, IEEE Transactions on, vol. 30 , no. 4, pp. 915 -927, April 2011.

[18] A. Marion, G. Forestier, H. Benoit-Cattin, S. Camarasu-Pop, P. Clarysse, R. da Silva, B. Gibaud, T. Glatard, P. Hugonnard, C. Lartizien, H. Liebgott, S. Specovius, J. Tabary, S. Valette, and D. Friboulet, "Multimodality medical image simulation of biological models with the virtual imaging platform (VIP)," in Computer-Based Medical Systems (CBMS), 2011 24th International Symposium on, June 2011, pp. 1 -6.

[19] W. Segars, D. Lalush, and B. Tsui, "A realistic spline-based dynamic heart phantom," Nuclear Science, IEEE Transactions on, vol. 46, no. 3, pp. $503-506$, June 1999.

[20] J. Montagnat and H. Delingette, "4D deformable models with temporal constraints : application to 4D cardiac image segmentation," Medical Image Analysis, vol. 9, no. 1, pp. 87-100, February 2005.

[21] J. A. Jensen, "FIELD: A program for simulating ultrasound systems," in 10th Nordic-Baltic Conference on Biomedical Imaging Published in Medical and Biological Engineering and Computing, vol. 34, 1996, pp. 351-353.

[22] M. J. Ledesma-Carbayo, J. Kybic, M. Desco, A. Santos, M. Sühling, P. Hunziker, and M. Unser, "Spatio-temporal nonrigid registration for ultrasound cardiac motion estimation." Medical Imaging, IEEE Transactions on, vol. 24, no. 9, pp. 1113-26, September 2005.

[23] H. Gao, H. F. Choi, P. Claus, S. Boonen, G. Van der Perre, W. Lauriks, and J. D'hooge, "A new convolution-based methodology to simulate ultrasound images in a 2D / 3D sector format," in Ultrasonics Symposium, 2007. IEEE, 2007, pp. $2243-2246$.

[24] T. Glatard, A. Marion, H. Benoit-Cattin, S. Camarasu-Pop, P. Clarysse, R. Ferreira da Silva, G. Forestier, B. Gibaud, C. Lartizien, H. Liebgott, K. Moulin, and D. Friboulet, "Multi-modality image simulation with the virtual imaging platform: Illustration on cardiac MRI and echography," in IEEE International Symposium on Biomedical Imaging (ISBI), Barcelona, Spain, 2012, pp. 98-101.

[25] R. Haddad, I. Magnin, and P. Clarysse, "A new fully-digital anthropomorphic and dynamic thorax/heart model," in Engineering in Medicine and Biology Society, 2007. EMBS 2007. 29th Annual International Conference of the IEEE, August 2007, pp. 5999 -6002.

[26] M. Sermesant, H. Delingette, and N. Ayache, "An electromechanical model of the heart for image analysis and simulation," Medical Imaging, IEEE Transactions on, vol. 25, pp. 612-625, 2006.

[27] M. Sermesant, R. Chabiniok, P. Chinchapatnam, T. Mansi, F. Billet, P. Moireau, J. Peyrat, K. Wong, J. Relan, K. Rhode, M. Ginks, P. Lambiase, H. Delingette, M. Sorine, C. Rinaldi, D. Chapelle, R. Razavi, and N. Ayache, "Patient-specific electromechanical models of the heart for the prediction of pacing acute effects in CRT: A preliminary clinical validation," Medical Image Analysis, vol. 16, no. 1, pp. 201-215, 2012.

[28] D. Chapelle, P. Le Tallec, P. Moireau, and M. Sorine, "An energypreserving muscle tissue model: formulation and compatible discretizations," International Journal for Multiscale Computational Engineering, vol. 10, no. 2, pp. 189-211, 2012.

[29] N. Smith, D. Nickerson, E. Crampin, and P. Hunter, "Computational mechanics of the heart. from tissue structure to ventricular function," Journal of Elasticity, vol. 61, no. 1, pp. 113-141, 2000.

[30] M. E. Belik, T. P. Usyk, and A. D. McCulloch, "Computational methods for cardiac electrophysiology," in Computational Models for the Human Body, ser. Handbook of Numerical Analysis, N. Ayache, Ed. Elsevier, 2004, vol. 12, pp. $129-187$.

[31] J. Sainte-Marie, D. Chapelle, R. Cimrman, and M. Sorine, "Modeling and estimation of the cardiac electromechanical activity," Computers and Structures, vol. 84, pp. 1743 - 1759, 2006.

[32] P. Shi and H. Liu, "Stochastic finite element framework for simultaneous estimation of cardiac kinematic functions and material parameters," Medical Image Analysis, vol. 7, no. 4, pp. 445-464, 2003.

[33] V. Y. Wang, H. I. Lam, D. B. Ennis, B. R. Cowan, A. A. Young, and M. P. Nash, "Modelling passive diastolic mechanics with quantitative MRI of cardiac structure and function," Medical Image Analysis, vol. 13, no. 5 , pp. 773-784, October 2009.

[34] H. Delingette, F. Billet, K. C. L. Wong, M. Sermesant, K. Rhode, M. Ginks, C. A. Rinaldi, R. Razavi, and N. Ayache, "Personalization of cardiac motion and contractility from images using variational data assimilation," Biomedical Engineering, IEEE Transactions on, vol. 59, no. 1, pp. $20-24$, January 2012.

[35] J. Xi, P. Lamata, J. Lee, P. Moireau, D. Chapelle, and N. Smith, "Myocardial transversely isotropic material parameter estimation from in-silico measurements based on reduced-order unscented Kalman filter," Journal of the Mechanical Behavior of Biomedical Materials, vol. 4, no. 7, pp. 1090-1102, 2011.

[36] R. Chabiniok, P. Moireau, P.-F. Lesault, A. Rahmouni, J.-F. Deux, and D. Chapelle, "Estimation of tissue contractility from cardiac cine-MRI using a biomechanical heart model," Biomechanics and Modeling in Mechanobiology, vol. 11, pp. 609-630, 2012.

[37] O. Camara, M. Schweiger, R. Scahill, W. Crum, B. Sneller, J. Schnabel, G. Ridgway, D. Cash, D. Hill, and N. Fox, "Phenomenological model of diffuse global and regional atrophy using finite-element methods," Medical Imaging, IEEE Transactions on, vol. 25, no. 11, pp. 1417 1430, November 2006

[38] P. Clarysse, J. Tafazzoli, P. Delachartre, and P. Croisille, "Simulation based evaluation of cardiac motion estimation methods in tagged-MR image sequences," Journal of Cardiovascular Magnetic Resonance, vol. 13, no. Suppl 1, p. P360, 2011.

[39] C. Tobon-Gomez, C. Butakoff, S. Aguade, F. Sukno, G. Moragas, and A. Frangi, "Automatic construction of 3D-ASM intensity models by simulating image acquisition: Application to myocardial gated SPECT studies," Medical Imaging, IEEE Transactions on, vol. 27, no. 11, pp. 1655 -1667, November 2008.

[40] N. Toussaint, T. Mansi, H. Delingette, N. Ayache, and M. Sermesant, "An integrated platform for dynamic cardiac simulation and image processing: Application to personalised tetralogy of fallot simulation," in Proc. Eurographics Workshop on Visual Computing for Biomedicine (VCBM), Delft, The Netherlands, 2008.

[41] L. Rineau, S. Tayeb, and M. Yvinec, "3D mesh generation," in CGAL User and Reference Manual, 3rd ed., CGAL Editorial Board, Ed., 2009.

[42] Q. Fang and D. A. Boas, "Tetrahedral mesh generation from volumetric binary and gray-scale images," in IEEE International Symposium on Biomedical Imaging (ISBI), 2009, pp. 1142-1145.

[43] M. Sermesant, E. Konukoglu, H. Delingette, Y. Coudiere, P. Chinchaptanam, K. Rhode, R. Razavi, and N. Ayache, "An anisotropic multi-front fast marching method for real-time simulation of cardiac electrophysiology," in Functional Imaging and Modeling of the Heart, ser. LNCS, F. B. Sachse and G. Seemann, Eds., vol. 4466. Springer Heidelberg, 7-9 June 2007, pp. 160-169. 
[44] S. Julier and J. Uhlmann, "A new extension of the Kalman filter to nonlinear systems," in International Symposium on Aerospace/Defense Sensing, Simulation and Controls, vol. 3, 1997, p. 26.

[45] S. Marchesseau, H. Delingette, M. Sermesant, K. Rhode, S. Duckett, C. Rinaldi, R. Razavi, and N. Ayache, "Cardiac mechanical parameter calibration based on the unscented transform," in Medical Image Computing and ComputerAssisted Intervention - MICCAI 2012, ser. LNCS. Springer, Heidelberg, October 2012, to appear. [Online]. Available: http://www-sop.inria.fr/asclepios/Publications/Stephanie.Marchesseau/MICCAI-2012-Marchesseau.pdf

[46] T. Vercauteren, X. Pennec, A. Perchant, and N. Ayache, "Symmetric logdomain diffeomorphic registration: A demons-based approach," in $\mathrm{Med}$ ical Image Computing and Computer-Assisted Intervention - MICCAI 2008, ser. LNCS, D. Metaxas, L. Axel, G. Fichtinger, and G. Székely, Eds., vol. 5241. New York, USA: Springer, Heidelberg, September 2008, pp. 754-761.

[47] F. Dru and T. Vercauteren, "An ITK implementation of the symmetric log-domain diffeomorphic demons algorithm," Insight Journal - 2009, May 2009.

[48] M. Bossa and S. Olmos, "A new algorithm for the computation of the group logarithm of diffeomorphisms," in 2nd MICCAI Workshop on Mathematical Foundations of Computational Anatomy, X. Pennec, Ed., New-York, October 2008.

[49] F. Faure, C. Duriez, H. Delingette, J. Allard, B. Gilles, S. Marchesseau, H. Talbot, H. Courtecuisse, G. Bousquet, I. Peterlik, and S. Cotin, "Sofa: A multi-model framework for interactive physical simulation," in Soft Tissue Biomechanical Modeling for Computer Assisted Surgery, ser. Studies in Mechanobiology, Tissue Engineering and Biomaterials, Y. Payan, Ed. Springer Heidelberg, 2012, vol. 11, pp. 283-321.

[50] OSIRIX. (2012) Dicom sample image sets. [Online]. Available: http://pubimage.hcuge.ch:8080/DATA/MAGIX.zip

[51] K. McLeod, A. Prakosa, T. Mansi, M. Sermesant, and X. Pennec, "An incompressible log-domain demons algorithm for tracking heart tissue," in Statistical Atlases and Computational Models of the Heart. Imaging and Modelling Challenges, ser. LNCS, O. Camara, E. Konukoglu, M. Pop, K. Rhode, M. Sermesant, and A. Young, Eds., vol. 7085. Toronto: Springer, Heidelberg, September 2012, pp. 55-67.

[52] A. Prakosa, M. Sermesant, H. Delingette, E. Saloux, P. Allain, P. Cathier, P. Etyngier, N. Villain, and N. Ayache, "Synthetic echocardiographic image sequences for cardiac inverse electro-kinematic learning," in Medical Image Computing and Computer-Assisted Intervention - MICCAI 2011 ser. LNCS, G. Fichtinger, A. Martel, and T. Peters, Eds., vol. 6891. Toronto, Canada: Springer, Heidelberg, September 2011, pp. 500-507.

[53] _ - "Non-invasive activation times estimation using 3D echocardiography," in Statistical Atlases and Computational Models of the Heart, ser. LNCS, O. Camara, M. Pop, K. Rhode, M. Sermesant, N. Smith, and A. Young, Eds., vol. 6364. Springer, Heidelberg, 2010, pp. 212-221. 